Supporting Information for

\title{
Gallium(III) Chloride-Catalyzed Formation of Eight-Membered Rings from Enynes Bearing a Cyclic Olefin
}

Soo Min Kim, Sang Ick Lee, and Young Keun Chung*

Intelligent Textile System Research Center, and Department of Chemistry,

College of natural Sciences, Seoul National University, Seoul 151-747, Korea 
Contents of supporting information

I. General (S3)

II. General procedures for the synthesis of cyclic enyne and representative procedure for $\mathrm{GaCl}_{3}$ catalyzed cycloisomerization (S3-S4)

III. Characterization of new compounds (S4-S14)

IV. NMR spectra for new compounds (S14-S41) 


\section{General}

Workup procedures were done in air. All solvents were dried and distilled according to standard methods before use. Liquid was transferred via syringe or canula. TLC plates were visualized by ultraviolet light and treatment with acidic p-anisaldehyde stain followed by gentle heating. Gallium(III) chloride was purchased from Aldrich Chemical Co. and were used as received. ${ }^{1} \mathrm{H}$ NMR and ${ }^{13} \mathrm{C}$ NMR spectra were obtained with a Bruker 300 spectrometer. Infrared spectra were recorded on a Shimadzu IR-470 spectrometer. High Resolution Mass spectrum was measured at the Korea Basic Science Institute (Daegu). Single crystal data for $\mathbf{1 b}$ was collected on an Enraf-Nonius CCD single crystal X-ray diffractometer at room temperature using graphite-monochromated MoK $\alpha$ radiation $(\lambda=0.71073 \AA)$. Structures were solved by direct methods using SHELXS-97 and refined by full-matrix least-squares with SHELXL-97. $\mathbf{6 a}^{1}, \mathbf{6 b}^{1}, \mathbf{1 2 a}^{1}$, $\mathbf{1 2} \mathbf{b}^{1}$, and $\mathbf{1 4 \mathbf { a } ^ { 2 }}$ were known compounds.

\section{General procedures for the synthesis of cyclic enyne and representative procedure for $\mathrm{GaCl}_{3}$ catalyzed cycloisomerization}

1) General procedures for the synthesis of cyclic enyne (1a).

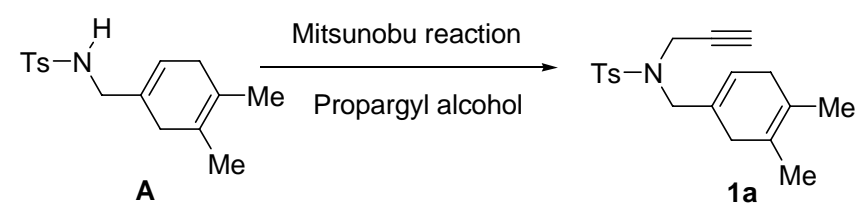

To a flame-dried $100 \mathrm{ml}$ schlenk capped with rubber septum, $15 \mathrm{ml}$ THF was injected via syringe under $\mathrm{N}_{2}$ flow. $0.9 \mathrm{~mL}$ (1.1 eq) of DIAD(Diisopropyl azodicarboxylate) and $1.2 \mathrm{~g}$ (1.2 eq) of $\mathrm{PPh}_{3}$ are added to the Schlenk. After $10 \mathrm{~min}, 1.3 \mathrm{~g}(4.46 \mathrm{mmol})$ of $\mathbf{A}^{2}$ and $0.3 \mathrm{~mL}(1.3 \mathrm{eq})$ of propargyl alcohol were added under $\mathrm{N}_{2}$. After reactant was disappeared, solvent was evaporated under reduced pressure. A flash column chromatography on a silica gel eluting with hexane and ethyl acetate (v/v, 10:1) gave the $1 \mathbf{a}$ in $92 \%$ yield.

\section{2) General procedure for $\mathrm{GaCl}_{3}$ catalyzed cycloisomerization of cyclic enyne.}

To a flame-dried $10 \mathrm{ml}$ Schlenk capped with rubber septum containing $5 \mathrm{~mL}$ of dry toluene, $14 \mathrm{mg}(10 \mathrm{~mol} \%)$ of $\mathrm{GaCl}_{3}$ and $0.7 \mathrm{mmol}$ of cyclic enyne were added sequentially. After the reactant was disappeared, $1 \mathrm{ml}$ diisopropylamine was added.

1. Mori, M.; Kuzuba, Y.; Kitamura, T.; Sato, Y. Org. Lett. 2002, 4, 3855.

2. Lee, S. I.; Park, S. Y; Park, J. H.; Jung, I. G.; Choi, S. Y.; Chung, Y. K.; Lee, B. Y. J. Org. Chem, 2006, $71,91$. 
After the reaction mixture was quenched, the solvent was removed under reduced pressure. A flash chromatography on a silica gel eluting with hexane and ethyl acetate $(\mathrm{v} / \mathrm{v}, 10: 1)$ gave the product.

\section{Characterization of New compounds}

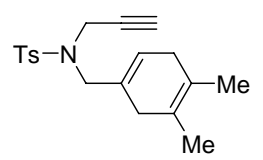

$N$-((4,5-dimethylcyclohexa-1,4-dienyl)methyl)-4-methyl- $N$-(prop-2-ynyl)benzenesulfonamide (1a)

${ }^{1}{ }_{\text {H NMR (CDCl }}, 300$ MHz) $\delta 1.63$ (s, $\left.3 \mathrm{H}\right), 1.64$ (s, $\left.3 \mathrm{H}\right), 1.94$ (t, $\left.1 \mathrm{H}, J=2.4 \mathrm{~Hz}\right)$, 2.43 (s, 3 H), 2.58 (m, 4 H), 3.72 (s, 2 H), 4.03 (d, 2 H, J = 2.4 Hz), 5.68 (s, 1 H), 7.29 $(\mathrm{d}, 2 \mathrm{H}, J=8.3 \mathrm{~Hz}), 7.74$ (d, $2 \mathrm{H}, J=8.3 \mathrm{~Hz}) \mathrm{ppm} ;{ }^{13} \mathbf{C} \mathbf{N M R}\left(\mathbf{C D C l}_{3}, \mathbf{7 5} \mathbf{M H z}\right) \delta 18.4$, 18.6, 21.8, 33.7, 33.9, 35.4, 52.1, 73.9, 76.6, 122.6, 123.3, 125.5, 128.0, 129.4, 129.6, 136.2, 143.6 ppm. Exact Mass for $\left(\mathrm{C}_{19} \mathrm{H}_{23} \mathrm{NO}_{2} \mathrm{~S}\right.$, EI): (calc) 329.1449, (found) 329.1447.

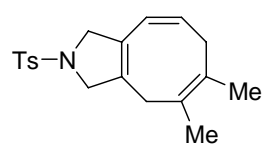

(5Z,8Z)-5,6-dimethyl-2-tosyl-2,3,4,7-tetrahydro-1H-cycloocta[c]pyrrole (1b)

${ }^{1} \mathbf{H}$ NMR (CDCl 3 , 300 MHz) $\delta 1.66$ (s, 3 H), 1.70 (s, 3 H), 2.44 (s, 3 H), 2.84 (s, 2 H), 2.88 (d, 2 H, $J=7.7 \mathrm{~Hz}$ ), 4.09 (m, $4 \mathrm{H}), 5.33$ (d, $1 \mathrm{H}, J=11.3 \mathrm{~Hz}), 5.89$ (td, $1 \mathrm{H}, J=7.7$ $\mathrm{Hz}, J=11.2 \mathrm{~Hz}), 7.33(\mathrm{~d}, 2 \mathrm{H}, J=8.1 \mathrm{~Hz}), 7.72(\mathrm{~d}, 2 \mathrm{H}, J=8.2 \mathrm{~Hz}) \mathrm{ppm} ;{ }^{13} \mathbf{C} \mathbf{~ N M R}$ $\left(\mathbf{C D C l}_{3}, \mathbf{7 5} \mathbf{M H z}\right) \delta 18.8,20.7,21.8,33.7,34.6,60.0,61.5,121.9,123.4,127.3,127.7$, 130.0, 131.4, 134.1, 134.9, 135.1, 143.7 ppm. Exact Mass for $\left(\mathrm{C}_{19} \mathrm{H}_{23} \mathrm{NO}_{2} \mathrm{~S}\right.$, EI): (calc) 329.1449, (found) 329.1447.

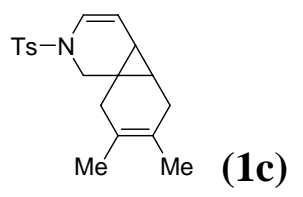

${ }^{1}$ H NMR (CDCl 3 , 300 MHz) $\delta 0.95(\mathrm{~m}, 1 \mathrm{H}), 1.03(\mathrm{~m}, 1 \mathrm{H}), 1.53(\mathrm{~s}, 6 \mathrm{H}), 2.17$ (m, 4 H), $2.42(\mathrm{~s}, 3 \mathrm{H}), 2.74$ (d, $1 \mathrm{H}, J=11.3 \mathrm{~Hz}), 3.83$ (d, $1 \mathrm{H}, J=11.3 \mathrm{~Hz}), 5.39$ (dd, $1 \mathrm{H}, J$ $=5.6 \mathrm{~Hz}, J=7.9 \mathrm{~Hz}), 6.27(\mathrm{~d}, 1 \mathrm{H}, J=7.9 \mathrm{~Hz}), 7.31(\mathrm{~d}, 2 \mathrm{H}, J=7.9 \mathrm{~Hz}), 7.65(\mathrm{~d}, 2 \mathrm{H}, J$ $=8.3 \mathrm{~Hz}) \mathrm{ppm} ;{ }^{13} \mathbf{C} \mathbf{N M R}\left(\mathbf{C D C l}_{3}, \mathbf{7 5} \mathbf{M H z}\right) \delta$ 16.8, 19.1, 19.3, 21.7, 15.8, 30.0, 30.9, 33.8, 45.7, 113.1, 120.3, 121.0, 122.5, 127.1, 129.9, 134.8, 143.8 ppm ; Exact Mass for 
$\left(\mathrm{C}_{19} \mathrm{H}_{23} \mathrm{NO}_{2} \mathrm{~S}, \mathrm{EI}\right):$ (calc) 329.1449, (found) 329.1451

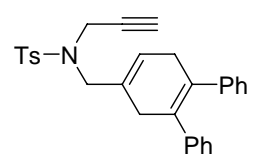

$N$-((4,5-diphenylcyclohexa-1,4-dienyl)methyl)-4-methyl- $N$-(prop-2-ynyl)benzenesulfonamide (2a)

${ }^{1}$ H NMR (CDCl 3 , 300 MHz) $\delta .00$ (s, $\left.1 \mathrm{H}\right), 2.40$ (s, $\left.3 \mathrm{H}\right), 3.11$ (m, $\left.4 \mathrm{H}\right), 3.84$ (s, $\left.2 \mathrm{H}\right)$, 4.14 (s, $2 \mathrm{H}), 5.86$ (s, $1 \mathrm{H}), 7.00(\mathrm{~m}, 4 \mathrm{H}), 7.09$ (m, $6 \mathrm{H}), 7.28(\mathrm{~d}, 2 \mathrm{H}, J=8.0 \mathrm{~Hz}), 7.75$ $(\mathrm{d}, 2 \mathrm{H}, J=8.0 \mathrm{~Hz}) \mathrm{ppm} ;{ }^{13} \mathbf{C} \mathbf{N M R}\left(\mathbf{C D C l}_{3}, \mathbf{7 5} \mathbf{M H z}\right) \delta 21.7,34.2,34.3,35.8,52.0$, 74.0, 74.2, 124.7, 126.4, 128.0, 128.1, 128.3, 129.0, 129.5, 129.6, 129.8, 130.0, 131.6, 132.1, 136.4, 142.3, 142.5, 143.7 ppm; Exact Mass for $\left(\mathrm{C}_{29} \mathrm{H}_{27} \mathrm{NO}_{2} \mathrm{~S}\right.$, EI) (calc) 453.1762, (found) 453.1764 .

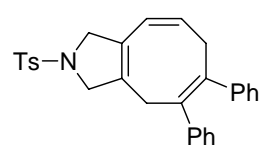

(5Z,8Z)-5,6-diphenyl-2-tosyl-2,3,4,7-tetrahydro-1H-cycloocta[c]pyrrole (2b)

$\left.{ }^{1} \mathbf{H ~ N M R ~ ( C D C l}_{3}, 300 \mathrm{MHz}\right) \delta 2.44(\mathrm{~s}, 3 \mathrm{H}), 3.39$ (m, $\left.4 \mathrm{H}\right), 4.14(\mathrm{~d}, 2 \mathrm{H}, J=16 \mathrm{~Hz})$, $4.15(\mathrm{~d}, 2 \mathrm{H}, J=16 \mathrm{~Hz}), 5.51(\mathrm{~d}, 1 \mathrm{H}, J=11.4 \mathrm{~Hz}), 5.89$ (td, $1 \mathrm{H}, J=7.6 \mathrm{~Hz}, J=11.1$ Hz), 6.83 (m, 2 H), 6.94 (m, 2 H), 7.05 (m, $6 \mathrm{H}), 7.32$ (d, 2 H, J = 7.9 Hz), 7.70 (d, 2 H, $J=8.0 \mathrm{~Hz}) \mathrm{ppm} ;{ }^{13} \mathbf{C} \mathbf{~ N M R}\left(\mathbf{C D C l}_{3}, \mathbf{7 5} \mathbf{M H z}\right) \delta 21.7,34.6,35.4,60.0,61.1,122.5$, 126.2, 126.3, 127.7, 127.9, 128.0, 128.4, 129.3, 129.6, 130.0, 132.1, 134.2, 134.3, 134.7, 139.2, 142.5, 143.61, 143.64 ppm; Exact Mass for $\left(\mathrm{C}_{29} \mathrm{H}_{27} \mathrm{NO}_{2} \mathrm{~S}\right.$, EI) (calc) 453.1762, (found) 453.1764 .

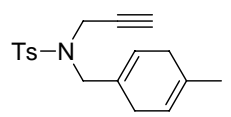

4-methyl- $N$-((4-methylcyclohexa-1,4-dienyl)methyl)- $N$-(prop-2-ynyl)benzenesulfonamide (3a)

${ }^{1}{ }_{\text {H NMR (CDCl }}, 300$ MHz) $\delta$ 1.66(s, $\left.3 \mathrm{H}\right), 1.98(\mathrm{~m}, 1 \mathrm{H}), 2.41(\mathrm{~s}, 3 \mathrm{H}), 2.61(\mathrm{~m}, 4 \mathrm{H})$, 3.72(s, 2 H), 4.02(s, 2 H), 5.42(m, 1 H), 5.70(m, 1 H), 7.28(d, 2 H, J=8.0 Hz), 7.72(d, 2 $\mathrm{H}, J=8.0 \mathrm{~Hz}) \mathrm{ppm} ;{ }^{13} \mathbf{C} \mathbf{~ N M R}\left(\mathbf{C D C l}_{3}, \mathbf{7 5} \mathbf{~ M H z}\right) \delta 21.5,23.2,27.7,31.6,35.3,52.2$, 73.8, 76.4, 118.4, 124.9, 127.8, 128.9, 129.4, 130.6, 136.0, 143.5 ppm; Exact Mass for $\left(\mathrm{C}_{18} \mathrm{H}_{21} \mathrm{NO}_{2} \mathrm{~S}, \mathrm{EI}\right)$ (calc) 315.1293, (found) 315.1296. 


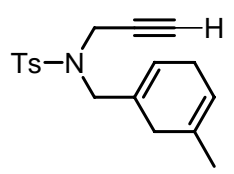

4-methyl- $N$-((5-methylcyclohexa-1,4-dienyl)methyl)- $N$-(prop-2-ynyl)benzenesulfonamide (3a')

${ }^{1} \mathbf{H}$ NMR (CDCl 3 , 300 MHz) $\delta 1.67(\mathrm{~s}, 3 \mathrm{H}), 2.02(\mathrm{~m}, 1 \mathrm{H}), 2.41(\mathrm{~s}, 3 \mathrm{H}), 2.50-2.56(\mathrm{~m}, 2$ H), 2.66-2.70(m, 2 H), 3.73(s, 2 H), 4.01(s, 2 H), 5.36(m, 1 H), 5.70(m, 1 H), 7.28(d, 2 $\mathrm{H}, J=8.0 \mathrm{~Hz}), 7.72(\mathrm{~d}, 2 \mathrm{H}, J=8.0 \mathrm{~Hz}) \mathrm{ppm} ;{ }^{13} \mathbf{C} \mathbf{N M R}\left(\mathbf{C D C l}_{3}, \mathbf{7 5} \mathbf{M H z}\right) \delta 21.5,23.2$, 27.7, 31.6, 35.2, 52.2, 73.8, 76.4, 117.8, 124.9, 127.8, 128.9, 129.4, 132.2, 136.0, 143.5 ppm; Exact Mass for $\left(\mathrm{C}_{18} \mathrm{H}_{21} \mathrm{NO}_{2} \mathrm{~S}, \mathrm{EI}\right)$ (calc) 315.1293, (found) 315.1296 .

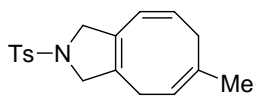

(5Z,8Z)-6-methyl-2-tosyl-2,3,4,7-tetrahydro-1H-cycloocta[c]pyrrole (3b)

${ }^{1} \mathbf{H}_{\text {NMR }}\left(\mathbf{C D C l}_{3}, 300 \mathrm{MHz}\right) \delta 1.65(\mathrm{~s}, 3 \mathrm{H}), 2.34(\mathrm{~s}, 3 \mathrm{H}), 2.59-2.69(\mathrm{~m}, 4 \mathrm{H}), 4.00(\mathrm{~m}, 4$ H), 5.00-5.10(m, $1 \mathrm{H}), 5.34-5.37(\mathrm{~m}, 1 \mathrm{H}), 5.75-5.79(\mathrm{~m}, 1 \mathrm{H}), 7.24(\mathrm{~d}, 2 \mathrm{H}, J=8.0 \mathrm{~Hz})$, 7.62(d, $2 \mathrm{H}, J=8.0 \mathrm{~Hz}) \mathrm{ppm} ;{ }^{13} \mathbf{C} \mathbf{N M R}\left(\mathbf{C D C l}_{3}, \mathbf{7 5} \mathbf{~ M H z}\right) \delta 21.7,23.9,26.3,32.3,59.5$, 60.5, 116.9, 122.1, 122.6, 127.58, 127.6, 129.9, 133.9, 134.7, 140.3, 143.6 ppm; Exact Mass for $\left(\mathrm{C}_{18} \mathrm{H}_{21} \mathrm{NO}_{2} \mathrm{~S}, \mathrm{EI}\right)$ (calc) 315.1293, (found) 315.1293<smiles>CC1CC=CC=C2CN(C)CC21</smiles>

(5Z,8Z)-5-methyl-2-tosyl-2,3,4,7-tetrahydro-1H-cycloocta[c]pyrrole (3b')

${ }^{1} \mathbf{H}$ NMR (CDCl 3 , 300 MHz) $\delta 1.61(\mathrm{~s}, 3 \mathrm{H}), 2.35(\mathrm{~s}, 3 \mathrm{H}), 2.59-2.65(\mathrm{~m}, 2 \mathrm{H}), 2.72-$ 2.74(m, 2 H), 4.00(m, 4 H), 5.34-5.41(m, 2 H), 5.70-5.74(m, 1 H), 7.24(d, 2 H, J=8.0 $\mathrm{Hz}), 7.62(\mathrm{~d}, 2 \mathrm{H}, J=8.0 \mathrm{~Hz}) \mathrm{ppm} ;{ }^{13} \mathbf{C}$ NMR (CDCl 3 , $\left.75 \mathbf{~ M H z}\right) \delta 21.7,25.4,27.6,31.2$, 59.1, 60.6, 116.9, 122.2, 122.5, 127.6, 127.7, 129.9, 133.5, 134.7, 140.3, 143.7 ppm; Exact Mass for $\left(\mathrm{C}_{18} \mathrm{H}_{21} \mathrm{NO}_{2} \mathrm{~S}, \mathrm{EI}\right)$ (calc) 315.1293, (found) 315.1293

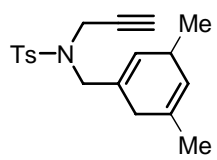

$N$-((3,5-dimethylcyclohexa-1,4-dienyl)methyl)-4-methyl- $N$-(prop-2-ynyl)benzenesulfonamide (4a)

${ }^{1} \mathbf{H ~ N M R ~}_{\left(\mathbf{C D C l}_{3}, 300 \mathrm{MHz}\right)} \delta 0.91(\mathrm{~d}, 3 \mathrm{H}, J=7.2 \mathrm{~Hz}), 1.58(\mathrm{~s}, 3 \mathrm{H}), 1.89(\mathrm{t}, 1 \mathrm{H}, J=$ 
$2.3 \mathrm{~Hz}), 2.31(\mathrm{~s}, 3 \mathrm{H}), 2.39(\mathrm{~s}, 1 \mathrm{H}), 2.40(\mathrm{~s}, 1 \mathrm{H}), 2.68(\mathrm{~m}, 1 \mathrm{H}), 3.64(\mathrm{~s}, 2 \mathrm{H}), 3.91(\mathrm{~d}$, $1 \mathrm{H}, J=2.6 \mathrm{~Hz}), 3.93(\mathrm{~d}, 1 \mathrm{H}, J=2.7 \mathrm{~Hz}), 5.19(\mathrm{~m}, 1 \mathrm{H}), 5.50(\mathrm{~m}, 1 \mathrm{H}), 7.19$ (d, $2 \mathrm{H}, J$ $=8.3 \mathrm{~Hz}), 7.64(\mathrm{~d}, 2 \mathrm{H}, J=8.3 \mathrm{~Hz}) \mathrm{ppm} ;{ }^{13} \mathbf{C} \mathbf{~ N M R}\left(\mathbf{C D C l}_{3}, \mathbf{7 5} \mathbf{M H z}\right) \delta 21.3,22.0$, 22.8, 31.6, 32.0, 35.1, 52.0, 73.8, 76.2, 124.3, 127.6, 127.7, 129.3, 129.8, 131.0, 135.9, 143.3 ppm; Exact Mass for $\left(\mathrm{C}_{19} \mathrm{H}_{23} \mathrm{NO}_{2} \mathrm{~S}, \mathrm{EI}\right)$ (calc) 329.1449 , (found) 329.1147

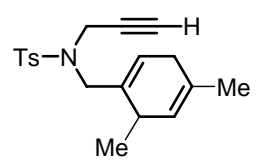

$N$-((4,6-dimethylcyclohexa-1,4-dienyl)methyl)-4-methyl- $N$-(prop-2-ynyl)benzenesulfonamide (4a')

${ }^{1}$ H NMR (CDCl 3 , 300 MHz) $\delta 1.02(\mathrm{~d}, 3 \mathrm{H}, J=7.0 \mathrm{~Hz}), 1.56(\mathrm{~s}, 3 \mathrm{H}), 1.86(\mathrm{t}, 1 \mathrm{H}, J=$ $2.4 \mathrm{~Hz}), 2.27$ (s, $3 \mathrm{H}), 2.35$, (m, $1 \mathrm{H}), 2.45$ (m, $2 \mathrm{H}), 3.70(\mathrm{~m}, 2 \mathrm{H}), 3.86(\mathrm{~d}, 1 \mathrm{H}, J=2.6$ $\mathrm{Hz}), 3.99,(\mathrm{~d}, 1 \mathrm{H}, J=2.4 \mathrm{~Hz}), 5.24,(\mathrm{~m}, 1 \mathrm{H}), 5.59$, (t, $1 \mathrm{H}, J=3.1 \mathrm{~Hz}), 7.19$ (d, $2 \mathrm{H}, J$ $=8.3 \mathrm{~Hz}), 7.64(\mathrm{~d}, 2 \mathrm{H}, J=8.3 \mathrm{~Hz}) \mathrm{ppm} ;{ }^{13} \mathbf{C} \mathbf{~ N M R}\left(\mathbf{C D C l}_{3}, \mathbf{7 5} \mathbf{M H z}\right) \delta 20.2,21.0$, 22.6, 30.9, 31.5, 35.3, 49.8, 73.9, 76.1, 125.1, 125.5, 126.3, 127.6, 129.2, 133.3, 135.7, 143.3 ppm; Exact Mass for $\left(\mathrm{C}_{19} \mathrm{H}_{23} \mathrm{NO}_{2} \mathrm{~S}, \mathrm{EI}\right)$ (calc) 329.1449 , (found) 329.1147

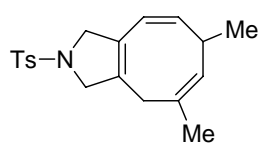

(5Z,8Z)-5,7-dimethyl-2-tosyl-2,3,4,7-tetrahydro-1H-cycloocta[c]pyrrole (4b)

${ }^{1} \mathbf{H ~ N M R ~}_{\left(\mathbf{C D C l}_{3}, 500 \mathrm{MHz}\right)} \delta 1.00(\mathrm{~d}, 3 \mathrm{H}, J=6.9 \mathrm{~Hz}), 1.64(\mathrm{~s}, 3 \mathrm{H}), 2.22(\mathrm{~d}, 1 \mathrm{H}, J=$ $16.8 \mathrm{~Hz}), 2.40(\mathrm{~s}, 3 \mathrm{H}), 3.31(\mathrm{~d}, 1 \mathrm{H}, J=16.6 \mathrm{~Hz}), 3.40(\mathrm{dd}, 1 \mathrm{H}, J=6.9 \mathrm{~Hz}, J=13.9$ Hz), 3.97 (m, $1 \mathrm{H}, \mathrm{J}=16.4 \mathrm{~Hz}), 4.03(\mathrm{~m}, 1 \mathrm{H}), 4.11(\mathrm{~m}, 1 \mathrm{H}), 4.21(\mathrm{~m}, 1 \mathrm{H}), 5.15$ (m, 1 H), $5.31(\mathrm{~d}, 1 \mathrm{H}, J=11.2 \mathrm{~Hz}), 5.43(\mathrm{dd}, 1 \mathrm{H}, J=6.3 \mathrm{~Hz}, J=11.2 \mathrm{~Hz}), 7.30$ (d, $2 \mathrm{H}, J=$ $8.5 \mathrm{~Hz}), 7.69$ (d, $2 \mathrm{H}, J=8.3 \mathrm{~Hz}) \mathrm{ppm} ;{ }^{13} \mathbf{C}$ NMR (CDCl $\left.\mathbf{3}, \mathbf{7 5} \mathbf{M H z}\right) \delta 20.7,21.7,25.4$, 32.0, 32.7, 59.6, 61.0, 119.6, 127.7, 128.3, 128.6, 129.9, 131.0, 133.1, 134.3, 141.7, $143.6 \mathrm{ppm}$; Exact Mass for $\left(\mathrm{C}_{19} \mathrm{H}_{23} \mathrm{NO}_{2} \mathrm{~S}, \mathrm{EI}\right)$ : (calc) 329.1449 , (found) 329.1447<smiles>Cc1cccc(CN(C)C)c1N(C)Cc1ccccc1</smiles>

\section{$\mathrm{N}$-(2,4-dimethylbenzyl)-4-methyl- $\mathrm{N}$-(prop-2-ynyl)benzenesulfonamide (4d)}

${ }^{1} \mathbf{H}$ NMR (CDCl 3,300 MHz) $\delta 2.00(t, 1 \mathrm{H}, J=2.4 \mathrm{~Hz}), 2.29$ (s, $\left.6 \mathrm{H}\right), 2.44(\mathrm{~s}, 3 \mathrm{H})$, 3.96 (d, $2 \mathrm{H}, J=2.5 \mathrm{~Hz}), 4.28$ (s, $2 \mathrm{H}), 6.94$ (s, $3 \mathrm{H}), 7.32$ (d, 2H, J = 8.0 Hz), 7.79 (d, 2 
$\mathrm{H}, J=8.3 \mathrm{~Hz}) \mathrm{ppm} ;{ }^{13} \mathbf{C}$ NMR (CDCl 3 , $\left.75 \mathbf{M H z}\right) \delta 21.4,21.8,35.7,49.9,74.2,76.5$, 126.7, 127.9, 128.0, 129.7, 129.8, 129.9, 134.9, 136.3, 138.4, 143.7 ppm ; Exact Mass for $\left(\mathrm{C}_{19} \mathrm{H}_{21} \mathrm{NO}_{2} \mathrm{~S}, \mathrm{EI}\right)$ : (calc) 327.1293, (found) 327.1293 .

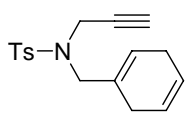

$N$-(cyclohexa-1,4-dienylmethyl)-4-methyl- $N$-(prop-2-ynyl)benzenesulfonamide (5a) ${ }^{1}{ }_{\text {H NMR (CDCl }}$, 300 MHz) $\delta$ 1.97(s, 1 H), 2.42 (s, 3 H), 2.68 (m, 4 H), 3.71 (s, 2 H), 4.04 (s, $2 \mathrm{H}), 5.71$ (m, $3 \mathrm{H}), 7.29$ (d, $2 \mathrm{H}, J=7.8 \mathrm{~Hz}), 7.74$ (d, $2 \mathrm{H}, J=7.8 \mathrm{~Hz}) \mathrm{ppm}$; ${ }^{13} \mathbf{C} \mathbf{~ N M R}\left(\mathbf{C D C l}_{3}\right.$, 75 MHz) $\delta 21.7,26.899,26.904,35.4,52.5,73.9,76.6,123.7,124.3$, 125.1, 127.9, 129.2, 129.6, 136.2, 143.6 ppm. Exact Mass for $\left(\mathrm{C}_{17} \mathrm{H}_{19} \mathrm{NO}_{2} \mathrm{~S}, \mathrm{EI}\right)$ : (calc) 301.1136, (found) 301.1137

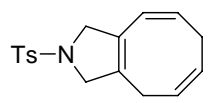

(4Z,7Z)-2-tosyl-2,3,6,9-tetrahydro-1H-cycloocta[c]pyrrole (5b)

${ }^{1}$ H NMR (CDCl 3 , 300 MHz) $\delta 2.43$ (s, 3 H), 2.74 (m, 4 H), 4.10 (s, 4 H), 5.43 (m, 1 H), $5.60(\mathrm{~d}, 1 \mathrm{H}, J=10.9 \mathrm{~Hz}), 5.71(\mathrm{~m}, 2 \mathrm{H}), 7.33$ (d, $2 \mathrm{H}, J=8.0 \mathrm{~Hz}), 7.72$ (d, $2 \mathrm{H}, J=8.1$ $\mathrm{Hz}) \mathrm{ppm} ;{ }^{13} \mathbf{C} \mathbf{N M R}\left(\mathbf{C D C l}_{3}, \mathbf{7 5} \mathbf{M H z}\right) \delta 21.7,26.0,27.2,58.5,59.7,122.9,123.3$, 127.6, 128.8 129.3, 129.9, 131.9, 132.0, 134.1, 143.6 ppm. Exact Mass for $\left(\mathrm{C}_{17} \mathrm{H}_{19} \mathrm{NO}_{2} \mathrm{~S}, \mathrm{EI}\right):$ (calc) 301.1136, (found) 301.1138

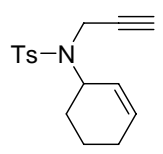

$N$-(cyclohex-2-enyl)-4-methyl- $N$-(prop-2-ynyl)benzenesulfonamide (7a)

${ }^{1}$ H NMR (CDCl 3 , 300 MHz) $\delta 1.54$ (m, 1 H), 1.77 (m, 3 H), 1.97 (m, 2 H), 2.19 (t, 1 H, $J=2.5 \mathrm{~Hz}), 2.41(\mathrm{~s}, 3 \mathrm{H}), 3.90(\mathrm{dd}, 1 \mathrm{H}, J=2.4 \mathrm{~Hz}, J=18.4 \mathrm{~Hz}), 4.12(\mathrm{dd}, 1 \mathrm{H}, J=2.5$ $\mathrm{Hz}, J=18.4 \mathrm{~Hz}), 4.47(\mathrm{~m}, 1 \mathrm{H}), 5.30(\mathrm{dd}, 1 \mathrm{H}, J=1.4 \mathrm{~Hz}, J=10.3 \mathrm{~Hz}), 5.87(\mathrm{~m}, 1 \mathrm{H})$, $7.28(\mathrm{~d}, 2 \mathrm{H}, J=8.1 \mathrm{~Hz}), 7.80(\mathrm{~d}, 2 \mathrm{H}, J=8.3 \mathrm{~Hz}) \mathrm{ppm} ;{ }^{13} \mathbf{C} \mathbf{N M R}\left(\mathbf{C D C l}_{3}, \mathbf{7 5} \mathbf{M H z}\right) \delta$ 21.4, 21.5, 24.3, 28.0, 32.7, 55.0, 72.1, 80.6, 127.0, 127.4, 129.5, 133.2, 138.0, 143.3 ppm; Exact Mass for $\left(\mathrm{C}_{16} \mathrm{H}_{19} \mathrm{NO}_{2} \mathrm{~S}, \mathrm{EI}\right)$ : (calc) 289.1136, (found) 289.1133.

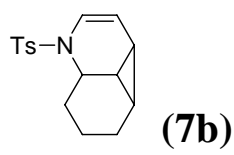


${ }^{1}{ }_{H}$ NMR (CDCl 3 , 300 MHz) $\delta 0.84(\mathrm{~m}, 1 \mathrm{H}), 1.05(\mathrm{~m}, 1 \mathrm{H}), 1.12(\mathrm{~m}, 1 \mathrm{H}), 1.29(\mathrm{~m}, 1$ H), $1.40(\mathrm{~m}, 1 \mathrm{H}), 1.46(\mathrm{~m}, 1 \mathrm{H}), 1.70(\mathrm{~m}, 1 \mathrm{H}), 1.78(\mathrm{~m}, 1 \mathrm{H}), 1.88(\mathrm{~m}, 1 \mathrm{H}), 2.41(\mathrm{~s}, 3$ H), $4.43(\mathrm{~m}, 1 \mathrm{H}), 5.30(\mathrm{dd}, 1 \mathrm{H} . J=3.9 \mathrm{~Hz}, J=7.9 \mathrm{~Hz}), 6.72(\mathrm{~d}, 1 \mathrm{H}, J=8.0 \mathrm{~Hz}), 7.28$ $(\mathrm{d}, 2 \mathrm{H}, J=7.9 \mathrm{~Hz}), 7.70$ (d, $2 \mathrm{H}, J=8.2 \mathrm{~Hz}) \mathrm{ppm} ;{ }^{13} \mathbf{C} \mathbf{~ N M R}\left(\mathbf{C D C l}_{3}, \mathbf{7 5} \mathbf{M H z}\right) \delta .6 .1$, 11.7, 13.4, 16.8, 19.3, 21.8, 31.5, 47.9, 108.9, 126.8, 126.9, 129.8, 137.2, 143.4 ppm; Exact Mass for $\left(\mathrm{C}_{16} \mathrm{H}_{19} \mathrm{NO}_{2} \mathrm{~S}, \mathrm{EI}\right)$ (calc) 289.1136, (found) 289.1135 .

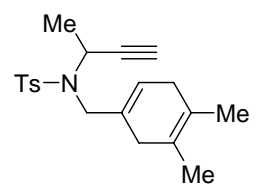

$N$-(but-3-yn-2-yl)- $N$-((4,5-dimethylcyclohexa-1,4-dienyl)methyl)-4-methylbenzenesulfonamide (8a)

${ }^{1} \mathbf{H}_{\text {NMR }}\left(\mathbf{C D C l}_{3}, 300 \mathrm{MHz}\right) \delta 1.36(\mathrm{~d}, 3 \mathrm{H}, J=7.1 \mathrm{~Hz}), 1.55(\mathrm{~s}, 3 \mathrm{H}), 1.56(\mathrm{~s}, 3 \mathrm{H})$, $2.06(\mathrm{~d}, 1 \mathrm{H}, J=2.5 \mathrm{~Hz}), 2.33$ (s, $3 \mathrm{H}), 2.53$ (m, $4 \mathrm{H}), 3.61$ (d, $1 \mathrm{H}, J=15.3 \mathrm{~Hz}), 3.83$ (d, $1 \mathrm{H}, J=15.2 \mathrm{~Hz}), 4.82(\mathrm{dq}, 1 \mathrm{H}, J=2.2 \mathrm{~Hz}, J=7.0 \mathrm{~Hz}), 5.62(\mathrm{~s}, 1 \mathrm{H}), 7.20$ (d, $2 \mathrm{H}, J=$ $8.2 \mathrm{~Hz}), 7.62(\mathrm{~d}, 2 \mathrm{H}, J=8.2 \mathrm{~Hz}) \mathrm{ppm} ;{ }^{13} \mathbf{C} \mathbf{~ N M R}\left(\mathbf{C D C l}_{3}, \mathbf{7 5} \mathbf{M H z}\right) \delta$ 18.3, 18.6, 21.7, 22.6, 33.9, 34.0, 46.5, 50.9, 73.7, 81.2, 122.6, 123.1, 123.3, 127.7, 130.0, 132.3, 136.7, 143.4 ppm; Exact Mass for $\left(\mathrm{C}_{20} \mathrm{H}_{25} \mathrm{NO}_{2} \mathrm{~S}\right.$, EI) (calc) 343.1606, (found) 343.1604.

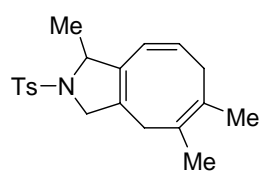

(5Z,8Z)-1,5,6-trimethyl-2-tosyl-2,3,4,7-tetrahydro-1H-cycloocta[c]pyrrole (8b)

${ }^{1} \mathbf{H}$ NMR (CDCl 3 , 300 MHz) $\delta 1.37$ (d, $3 \mathrm{H}, J=6.3 \mathrm{~Hz}$ ), 1.61 (s, $\left.3 \mathrm{H}\right), 1.68$ (s, $\left.3 \mathrm{H}\right)$, $2.42(\mathrm{~s}, 3 \mathrm{H}), 2.65(\mathrm{~d}, 1 \mathrm{H}, J=12.2 \mathrm{~Hz}), 2.67(\mathrm{~d}, 1 \mathrm{H}, J=12.7 \mathrm{~Hz}), 2.97(\mathrm{dd}, 2 \mathrm{H}, J=$ $7.8 \mathrm{~Hz}, J=13.9 \mathrm{~Hz}), 4.02(\mathrm{~d}, 1 \mathrm{H}, J=15.0 \mathrm{~Hz}), 4.14(\mathrm{dd}, 1 \mathrm{H}, J=4.4 \mathrm{~Hz}, J=15.2 \mathrm{~Hz})$, 4.35 (q, $1 \mathrm{H}, J=6.2 \mathrm{~Hz}), 5.34$ (d, $1 \mathrm{H}, J=11.1 \mathrm{~Hz}), 5.87$ (td, $1 \mathrm{H}, J=7.6 \mathrm{~Hz}, J=11.1$ $\mathrm{Hz}), 7.29$ (d, $2 \mathrm{H}, J=8.2 \mathrm{~Hz}), 7.70$ (d, $2 \mathrm{H}, J=8.2 \mathrm{~Hz}) \mathrm{ppm} ;{ }^{13} \mathbf{C} \mathbf{N M R}\left(\mathbf{C D C l}_{3}, 75\right.$ MHz) $\delta$ 19.2, 20.8, 21.7, 22.3, 33.9, 34.9, 60.4, 67.2, 122.3, 123.4, 127.6, 129.8, 130.9, 132.8, 134.0, 134.6, 135.3, 143.5 ppm; Exact Mass for $\left(\mathrm{C}_{20} \mathrm{H}_{25} \mathrm{NO}_{2} \mathrm{~S}\right.$, EI) (calc) 343.1606, (found) 343.1601 .

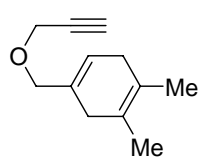

1,2-dimethyl-4-((prop-2-ynyloxy)methyl)cyclohexa-1,4-diene (9a) 
${ }^{1} \mathbf{H}$ NMR (CDCl 3 , 300 MHz) $\delta 1.56$ (s, $\left.3 \mathrm{H}\right), 1.58$ (s, $\left.3 \mathrm{H}\right), 2.34$ (t, $\left.1 \mathrm{H}, J=2.4 \mathrm{~Hz}\right)$, 2.52 (m, $4 \mathrm{H}), 3.89$ (s, $2 \mathrm{H}), 4.03$ (d, $2 \mathrm{H}, J=2.5 \mathrm{~Hz}) 5.64$ (s, $1 \mathrm{H}) \mathrm{ppm} ;{ }^{13} \mathbf{C}$ NMR $\mathbf{( C D C l}_{3}, 75$ MHz) o. 18.4, 18.6, 33.7, 33.9, 56.7, 74.3, 80.2, 122.8, 123.1, 123.8, 131.9 ppm; Exact Mass for $\left(\mathrm{C}_{12} \mathrm{H}_{16} \mathrm{O}, \mathrm{EI}\right)$ (calc) 176.1201, (found) 176.1202.

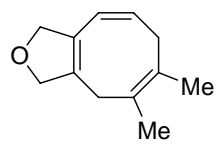

(5Z,8Z)-5,6-dimethyl-1,3,4,7-tetrahydrocycloocta[c]furan (9b)

${ }^{1} \mathbf{H}$ NMR (CDCl 3 , 300 MHz) $\delta 1.73$ (s, 3 H), 1.75 (s, 3 H), 2.91 (s, 2 H), 2.99 (d, 2 H, J $=7.7 \mathrm{~Hz}), 4.64(\mathrm{~s}, 4 \mathrm{H}), 5.43(\mathrm{~d}, 1 \mathrm{H}, J=11.0 \mathrm{~Hz}), 5.93(\mathrm{td}, 1 \mathrm{H}, J=7.7 \mathrm{~Hz}, J=10.9 \mathrm{~Hz})$ ppm; ${ }^{13} \mathbf{C}$ NMR (CDCl 3 , 75 MHz) $\delta$ 18.5, 20.8, 32.1, 34.7, 81.2, 82.5, 120.9, 123.4, 128.0, 131.6, 134.1, 136.4 ppm; Exact Mass for $\left(\mathrm{C}_{12} \mathrm{H}_{16} \mathrm{O}, \mathrm{EI}\right)$ (calc) 176.1201, (found) 176.1204 .

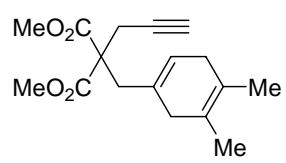

dimethyl 2-((4,5-dimethylcyclohexa-1,4-dienyl)methyl)-2-(prop-2-ynyl) malonate (10a)

v(CO) : $1726 \mathrm{~cm}^{-1} ;{ }^{1} \mathbf{H}$ NMR (CDCl 3 , 300 MHz) $\delta 1.50(\mathrm{~s}, 3 \mathrm{H}), 1.52(\mathrm{~s}, 3 \mathrm{H}), 1.98$ (t, $1 \mathrm{H}, J=2.5 \mathrm{~Hz}), 2.29$ (t, $2 \mathrm{H}, J=7.2 \mathrm{~Hz}), 2.52$ (s, $2 \mathrm{H}), 2.71(\mathrm{~s}, 2 \mathrm{H}), 2.73$ (d, $2 \mathrm{H}, J=$ $2.6 \mathrm{~Hz}), 3.65$ (s, $6 \mathrm{H}), 5.49$ (s, 1H) ppm; ${ }^{13} \mathbf{C}$ NMR (CDCl, 75 MHz) $\delta .18 .1,18.4,22.7$, 33.8, 36.6, 39.0, 52.7, 56.5, 71.8, 78.3, 122.6, 122.7, 125.0, 129.5, 170.6 ppm; Exact Mass for $\left(\mathrm{C}_{17} \mathrm{H}_{22} \mathrm{O}_{4}\right.$, EI) (calc) 290.1518, (found) 290.1521 .

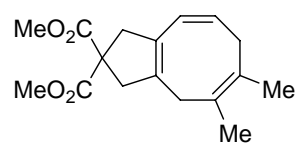

(5Z,8Z)-dimethyl 5,6-dimethyl-1H-cyclopenta[8]annulene-2,2(3H,4H,7H)-dicarboxylate (10b)

$v(\mathbf{C O}): 1726 \mathrm{~cm}^{-1} ;{ }^{1} \mathbf{H}$ NMR (CDCl 3 , 300 MHz) $\delta 1.72$ (s, $\left.6 \mathrm{H}\right), 2.91$ (d, $2 \mathrm{H}, J=7.2$ Hz), 2.95 (s, 2 H), 3.08 (s, 2 H), 3.11 (s, 2 H), 3.73 (s, $6 \mathrm{H}), 5.51$ (d, $1 \mathrm{H}, J=11.3 \mathrm{~Hz}$ ), $5.81(\mathrm{td}, 1 \mathrm{H}, J=7.5 \mathrm{~Hz}, J=11.2 \mathrm{~Hz}) \mathrm{ppm} ;{ }^{13} \mathbf{C} \mathbf{N M R}\left(\mathbf{C D C l}_{3}, \mathbf{7 5} \mathbf{M H z}\right) \delta 19.2,20.9$, 34.9, 36.2, 47.1, 49.1, 52.9, 53.0, 57.1, 124.5, 125.2, 129.2, 130.1, 132.7, 138.0, 172.8 ppm; Exact Mass for $\left(\mathrm{C}_{17} \mathrm{H}_{22} \mathrm{O}_{4}, \mathrm{EI}\right)$ (calc) 290.1518, (found) 290.1515 . 


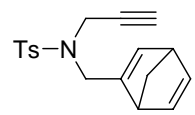

$N$-(bicyclo[2.2.1]hepta-2,5-dien-2-ylmethyl)-4-methyl- $N$-(prop-2-ynyl)benzenesulfonamide (11a)

${ }^{1} \mathbf{H}$ NMR (CDCl $\left.3,300 \mathrm{MHz}\right) \oint 1.94(\mathrm{t}, 1 \mathrm{H}, J=3.6 \mathrm{~Hz}), 2.01(\mathrm{~m}, 2 \mathrm{H}), 2.40(\mathrm{~s}, 3 \mathrm{H})$, $3.44(\mathrm{~s}, 1 \mathrm{H}), 3.54(\mathrm{~s}, 1 \mathrm{H}), 3.71(\mathrm{dd}, 1 \mathrm{H}, J=2.0 \mathrm{~Hz}, J=18.3 \mathrm{~Hz}), 3.86(\mathrm{~d}, 1 \mathrm{H}, J=$ $14.0 \mathrm{~Hz}), 4.03(\mathrm{dd}, 1 \mathrm{H}, J=1.8 \mathrm{~Hz}, J=18.6 \mathrm{~Hz}), 4.04(\mathrm{~d}, 1 \mathrm{H}, J=13.3 \mathrm{~Hz}), 6.50$ (s, 1 H), $6.69(\mathrm{~m}, 1 \mathrm{H}), 6.78(\mathrm{~m}, 1 \mathrm{H}), 7.28(\mathrm{~d}, 2 \mathrm{H}, J=8.1 \mathrm{~Hz}), 7.73(\mathrm{~d}, 2 \mathrm{H}, J=8.2 \mathrm{~Hz})$ ppm ; ${ }^{13} \mathbf{C}$ NMR (CDCl 3 , 75 MHz) $\delta 21.4,35.4,46.2,50.4,51.7,73.8,73.9,76.2$, 127.7, 129.3, 135.9, 141.6, 142.5, 142.8, 143.4, $151.5 \mathrm{ppm}$; Exact Mass for $\left(\mathrm{C}_{18} \mathrm{H}_{19} \mathrm{NO}_{2} \mathrm{~S}, \mathrm{EI}\right)$ : (calc) 313.1136, (found) 313.1134

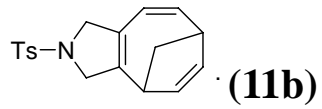

${ }^{1} \mathbf{H}$ NMR (CDCl 3 , 300 MHz) $\delta 1.38(\mathrm{~d}, 1 \mathrm{H}, J=11.5 \mathrm{~Hz}), 1.99(\mathrm{td}, 1 \mathrm{H}, J=6.6 \mathrm{~Hz}, J=$ $12.6 \mathrm{~Hz}), 2.43$ (s, $3 \mathrm{H}), 2.99(\mathrm{dd}, 1 \mathrm{H}, J=2.3 \mathrm{~Hz}, J=6.4 \mathrm{~Hz}), 3.13(\mathrm{dt}, 1 \mathrm{H}, J=2.6 \mathrm{~Hz}$, $J=7.2 \mathrm{~Hz}), 4.13(\mathrm{~m}, 1 \mathrm{H}), 4.21(\mathrm{~m}, 1 \mathrm{H}), 4.32(\mathrm{dd}, 2 \mathrm{H}, J=3.6 \mathrm{~Hz}, J=7.9 \mathrm{~Hz}), 5.20$ $(\mathrm{dd}, 1 \mathrm{H}, J=2.1 \mathrm{~Hz}, J=5.4 \mathrm{~Hz}), 5.28(\mathrm{dd}, 1 \mathrm{H}, J=2.4 \mathrm{~Hz}, J=5.4 \mathrm{~Hz}), 5.65$ (d, $1 \mathrm{H}, J$ $=11.2 \mathrm{~Hz}), 6.11(\mathrm{dd}, 1 \mathrm{H}, J=7.5 \mathrm{~Hz}, J=11.1 \mathrm{~Hz}), 7.32(\mathrm{~d}, 2 \mathrm{H}, J=8.3 \mathrm{~Hz}), 7.72(\mathrm{~d}, 2$ $\mathrm{H}, J=8.1 \mathrm{~Hz}) \mathrm{ppm} ;{ }^{13} \mathbf{C} \mathbf{N M R}\left(\mathbf{C D C l}_{3}, \mathbf{7 5} \mathbf{M H z}\right) \delta 21.7,30.2,41.2,43.1,58.1,58.3$, 120.0, 123.6, 124.0, 126.5, 127.7, 130.0, 134.4, 138.0, 140.6, 134.6 ppm; Exact Mass for $\left(\mathrm{C}_{18} \mathrm{H}_{19} \mathrm{NO}_{2} \mathrm{~S}, \mathrm{EI}\right)$ : (calc) 313.1136, (found) 313.1137 .

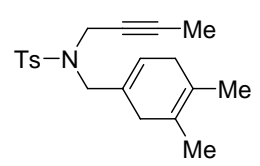

$N$-(but-2-ynyl)- $N$-((4,5-dimethylcyclohexa-1,4-dienyl)methyl)-4-methylbenzenesulfonamide (13a)

${ }^{1} \mathbf{H}$ NMR (CDCl 3,300 MHz) $\delta 1.49$ (t, $\left.3 \mathrm{H}, J=2.1 \mathrm{~Hz}\right), 1.61(\mathrm{~s}, 3 \mathrm{H}), 1.63(\mathrm{~s}, 3 \mathrm{H})$, 2.41 (s, $3 \mathrm{H}), 2.58$ (m, $4 \mathrm{H}), 3.67$ (s, $2 \mathrm{H}), 3.94$ (d, $2 \mathrm{H}, J=2.1 \mathrm{~Hz}), 5.64$ (s, $1 \mathrm{H}), 7.28$ $(\mathrm{d}, 2 \mathrm{H}, J=8.1 \mathrm{~Hz}), 7.73(\mathrm{~d}, 2 \mathrm{H}, J=8.1 \mathrm{~Hz}) \mathrm{ppm} ;{ }^{13} \mathbf{C} \mathbf{N M R}\left(\mathbf{C D C l}_{3}, \mathbf{7 5} \mathbf{M H z}\right) \delta 3.4$, 18.3, 18.6, 21.7, 33.8, 33.9, 36.0, 52.2, 71.8, 81.6, 122.5, 123.3, 125.0, 128.1, 129.3, 129.7, 136.4, 143.3 ppm. Exact Mass for $\left(\mathrm{C}_{20} \mathrm{H}_{25} \mathrm{~N}_{1} \mathrm{~S}_{1} \mathrm{O}_{2}\right.$, EI): (calc) 343.1606, (found) 343.1610 . 


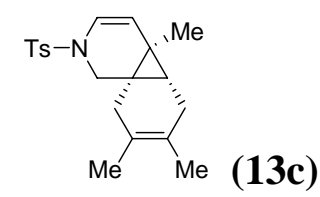

${ }^{1}$ H NMR (CDCl 3 , 300 MHz) $\delta 0.81(\mathrm{~s}, 3 \mathrm{H}), 0.89(\mathrm{~d}, \mathrm{~J}=7.2 \mathrm{~Hz}, 1 \mathrm{H}), 1.54(\mathrm{~s}, 3 \mathrm{H})$, $1.55(\mathrm{~s}, 3 \mathrm{H}), 1.88(\mathrm{~m}, 1 \mathrm{H}), 1.96(\mathrm{~m}, 2 \mathrm{H}), 2.12(\mathrm{~m}, 1 \mathrm{H}), 2.42(\mathrm{~s}, 3 \mathrm{H}), 2.76(\mathrm{~d}, \mathrm{~J}=11.3$ $\mathrm{Hz}, 1 \mathrm{H}), 3.80$ (d, J = 11.3 Hz, $1 \mathrm{H}), 5.17$ (d, J = 7.9 Hz, $1 \mathrm{H}), 6.22$ (d, J = 7.9 Hz, $1 \mathrm{H})$, $7.31(\mathrm{~d}, \mathrm{~J}=7.9 \mathrm{~Hz}, 2 \mathrm{H}), 7.64$ (d, J $=7.8 \mathrm{~Hz}, 2 \mathrm{H}) \mathrm{ppm} ;{ }^{13} \mathbf{C} \mathbf{N M R}\left(\mathbf{C D C l}_{3}, \mathbf{7 5} \mathbf{M H z}\right) \delta$ $12.1,18.7,18.9,19.3,21.7,25.4,27.8,29.7,31.1,46.1,119.3,120.0,122.6,124.2$, 127.2, 129.9, 135.1, 143.7 ppm. Exact Mass for $\left(\mathrm{C}_{20} \mathrm{H}_{25} \mathrm{~N}_{1} \mathrm{~S}_{1} \mathrm{O}_{2}\right.$, EI): (calc) 343.1606, (found) 343.1610 .

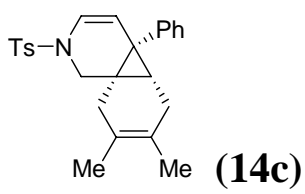

${ }^{1} \mathbf{H}$ NMR (CDCl 3 , 300 MHz) $\delta 1.03$ (s, $\left.3 \mathrm{H}\right), 1.08$ (s, $\left.3 \mathrm{H}\right), 1.33(\mathrm{~d}, \mathrm{~J}=4.7 \mathrm{~Hz}, 1 \mathrm{H})$, $2.16(\mathrm{~m}, 4 \mathrm{H}), 2.45$ (s, $3 \mathrm{H}), 2.94(\mathrm{~d}, \mathrm{~J}=11.5 \mathrm{~Hz}, 1 \mathrm{H}), 3.93$ (d, J = 11.5 Hz, $1 \mathrm{H}), 5.16$ $(\mathrm{d}, \mathrm{J}=7.9 \mathrm{~Hz}, 1 \mathrm{H}), 6.28(\mathrm{~d}, \mathrm{~J}=7.9 \mathrm{~Hz}, 1 \mathrm{H}), 7.00(\mathrm{~d}, \mathrm{~J}=7.8 \mathrm{~Hz}, 2 \mathrm{H}), 7.15(\mathrm{~m}, 2 \mathrm{H})$, 7.35 (m, $3 \mathrm{H}), 7.69$ (d, J = 7.8 Hz, $1 \mathrm{H}) \mathrm{ppm} ;{ }^{13} \mathbf{C} \mathbf{~ N M R}\left(\mathbf{C D C l}_{3}, \mathbf{7 5} \mathbf{M H z}\right) \delta 18.4,21.6$, 27.6, 29.5, 29.8, 32.7, 34.0, 46.0, 119.0, 119.2, 122.2, 123.5, 126.1, 127.1, 127.5, 127.8, 129.9, 134.9, 140.5, 143.7 ppm. Exact Mass for $\left(\mathrm{C}_{25} \mathrm{H}_{27} \mathrm{~S}_{1} \mathrm{O}_{2} \mathrm{~N}_{1}\right.$, EI): calcd 393.1762, obsd 393.1764

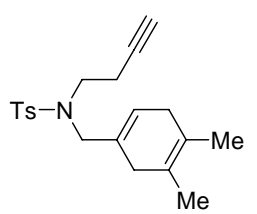

$N$-(but-3-ynyl)- $N$-((4,5-dimethylcyclohexa-1,4-dienyl)methyl)-4-methylbenzenesulfonamide (15a)

${ }^{1} \mathbf{H}$ NMR (CDCl 3,300 MHz) $\delta 1.60$ (s, $\left.3 \mathrm{H}\right), 1.62$ (s, $\left.3 \mathrm{H}\right), 1.93$ (t, $\left.1 \mathrm{H}, J=2.7 \mathrm{~Hz}\right)$, $2.40(\mathrm{~m}, 8 \mathrm{H}), 2.60$ (d, $2 \mathrm{H}, J=6.7 \mathrm{~Hz}), 3.25(\mathrm{~m}, 2 \mathrm{H}), 3.71(\mathrm{~s}, 2 \mathrm{H}), 5.60$ (m, $1 \mathrm{H}), 7.30$ $(\mathrm{d}, 2 \mathrm{H}, J=8.1 \mathrm{~Hz}), 7.70$ (d, $2 \mathrm{H}, J=8.3 \mathrm{~Hz}) \mathrm{ppm} ;{ }^{13} \mathbf{C} \mathbf{N M R}\left(\mathbf{C D C l}_{3}, \mathbf{7 5} \mathbf{M H z}\right) \delta .14 .4$, 18.3, 18.6, 19.2, 21.7, 33.9, 46.5, 54.9, 70.2, 81.3, 122.6, 123.0, 124.7, 127.3, 129.8, 130.8, 137.1, 143.5 ppm; Exact Mass for $\left(\mathrm{C}_{20} \mathrm{H}_{25} \mathrm{NO}_{2} \mathrm{~S}\right.$, EI) (calc) 343.1606, (found) 343.1605 . 


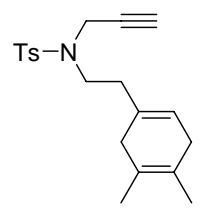

$N$-(2-(4,5-dimethylcyclohexa-1,4-dienyl)ethyl)-4-methyl- $N$-(prop-2-ynyl)benzenesulfonamide (16a)

${ }^{1}{ }_{\text {H NMR (CDCl }}, 300$ MHz) $\delta 1.63(\mathrm{~s}, 6 \mathrm{H}), 2.04$ (t, $\left.1 \mathrm{H}, J=2.4 \mathrm{~Hz}\right), 2.25$ (m, $\left.2 \mathrm{H}\right)$, 2.41 (s, 3 H), 2.54 (m, 4 H), 3.30 (m, 2 H), 4.15 (d, 2 H, J = 2.4 Hz), 5.47 (m, 1 H), 7.28 $(\mathrm{d}, 2 \mathrm{H}, J=8.0 \mathrm{~Hz}), 7.72(\mathrm{~d}, 2 \mathrm{H}, J=8.3 \mathrm{~Hz}) \mathrm{ppm} ;{ }^{13} \mathbf{C} \mathbf{N M R}\left(\mathbf{C D C l}_{3}, 75 \mathbf{M H z}\right) \delta 18.4$, 18.6, 21.7, 33.9, 35.3, 35.8, 36.4, 44.8, 73.9, 76.7, 121.5, 123.0, 123.1, 127.9, 129.6, 131.2, 136.0, 143.6 ppm; Exact Mass for $\left(\mathrm{C}_{20} \mathrm{H}_{25} \mathrm{NO}_{2} \mathrm{~S}\right.$, EI) (calc) 343.1606, (found) 343.1605 .

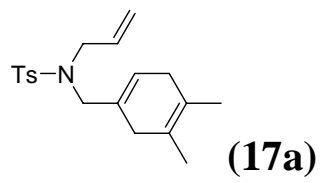

$N$-allyl- $N$-((4,5-dimethylcyclohexa-1,4-dienyl)methyl)-4-methylbenzenesulfonamide $\left.{ }^{1} \mathbf{H ~ N M R ~ ( C D C l}_{3}, 300 \mathrm{MHz}\right) \delta 1.61$ (s, $\left.6 \mathrm{H}\right), 2.42$ (m, $\left.5 \mathrm{H}\right), 2.60$ (s, $\left.2 \mathrm{H}\right), 3.70$ (s, $\left.2 \mathrm{H}\right)$, $3.76(\mathrm{~d}, 2 \mathrm{H}, J=6.3 \mathrm{~Hz}), 5.05$ (d, $1 \mathrm{H}, J=4.0 \mathrm{~Hz}), 5.10(\mathrm{~m}, 1 \mathrm{H}), 5.52(\mathrm{~m}, 2 \mathrm{H}), 7.29$ (d, $2 \mathrm{H}, J=8.0 \mathrm{~Hz}), 7.70(\mathrm{~d}, 2 \mathrm{H}, J=8.1 \mathrm{~Hz}) \mathrm{ppm} ;{ }^{13} \mathbf{C} \mathbf{N M R}\left(\mathbf{C D C l}_{3}, \mathbf{7 5} \mathbf{M H z}\right) \delta 18.3$, 18.6, 21.7, 33.86, 33.90, 49.2, 52.6, 119.1, 122.6, 123.2, 124.4, 127.4, 129.7, 130.3, 132.7, 137.9, 143.2 ppm; Exact Mass for $\left(\mathrm{C}_{19} \mathrm{H}_{25} \mathrm{NO}_{2} \mathrm{~S}\right.$, EI) (calc) 331.1606, (found) 331.1610 .

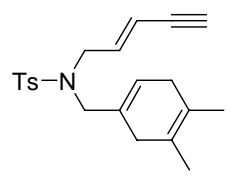

(E)- $N$-((4,5-dimethylcyclohexa-1,4-dienyl)methyl)-4-methyl- $N$-(pent-2-en-4ynyl)benzenesulfonamide (18a)

${ }^{1} \mathbf{H}$ NMR (CDCl 3 , 300 MHz) $\delta 1.53$ (s, $\left.6 \mathrm{H}\right), 1.97$ (s, $\left.1 \mathrm{H}\right), 2.34$ (m, $\left.5 \mathrm{H}\right), 2.52$ (s, $\left.2 \mathrm{H}\right)$, $3.61(\mathrm{~s}, 2 \mathrm{H}), 3.72(\mathrm{~d}, 2 \mathrm{H}, J=6.6 \mathrm{~Hz}), 5.41$ (d, $1 \mathrm{H}, J=16.0 \mathrm{~Hz}), 5.48(\mathrm{~s}, 1 \mathrm{H}), 5.81$ (td, $1 \mathrm{H}, J=6.7 \mathrm{~Hz}, J=15.7 \mathrm{~Hz}), 7.23(\mathrm{~d}, 2 \mathrm{H}, J=7.9 \mathrm{~Hz}), 7.62(\mathrm{~d}, 2 \mathrm{H}, J=8.2 \mathrm{~Hz}) \mathrm{ppm}$; ${ }^{13} \mathbf{C}$ NMR (CDCl 3 , 75 MHz) $\delta 14.3,18.3,21.6,33.8,47.9,53.0,60.5,78.4,81.2,112.8$, $122.5,123.0,125.0,127.3,129.8,129.9,137.3,139.3,143.5$ ppm; Exact Mass for $\left(\mathrm{C}_{21} \mathrm{H}_{25} \mathrm{NO}_{2} \mathrm{~S}, \mathrm{EI}\right)$ (calc) 355.1606, (found) 355.1606 . 


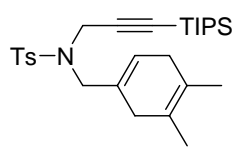

$N$-((4,5-dimethylcyclohexa-1,4-dienyl)methyl)-4-methyl- $N$-(3-(triisopropylsilyl)prop-2-ynyl)benzenesulfonamide (19a)

${ }^{1}$ H NMR (CDCl 3 , 300 MHz) $\delta 0.91$ (m, 21 H), 1.63 (s, 3 H), 1.66 (s, 3 H), 2.39 (s, 3 H), 2.61 (s, 4 H), 3.78 (s, 2 H), 4.13 (s, 2 H), 5.68 (s, 1 H), 7.26 (d, 2 H, J = 8.0 Hz), 7.73 (d, $2 \mathrm{H}, J=8.3 \mathrm{~Hz}) \mathrm{ppm} ;{ }^{13} \mathbf{C} \mathbf{N M R}\left(\mathbf{C D C l}_{3}, 75 \mathbf{M H z}\right) \delta$ 11.1, 18.2, 18.5, 21.5, 33.6, 33.8, 36.3, 51.7, 87.0, 99.8, 122.4, 123.2, 125.3, 127.6, 129.3, 129.7, 136.5, 143.2 ppm; Exact Mass for $\left(\mathrm{C}_{28} \mathrm{H}_{43} \mathrm{NO}_{2} \mathrm{~S}_{1} \mathrm{Si}_{1}\right.$, EI) (calc) 485.2784, (found) 485.2782. 